\title{
Recollements arising from cotorsion pairs on extriangulated categories ${ }^{\star}$
}

\author{
Yonggang $\mathrm{Hu}$ and Panyue Zhou
}

\begin{abstract}
This paper is devoted to constructing some recollements of additive categories associated to concentric twin cotorsion pairs on an extriangulated category. As an application, this result generalizes the work by Chen-Liu-Yang in a triangulated case. Moreover, it highlights new phenomena when it applied to an exact category. Finally, we give some applications to illustrate our main results. In particular, we obtain the Krause's recollement whose the proofs are both elementary and very general.
\end{abstract}

Key words: extriangulated categories; recollements; cotorsion pairs; adjoint pairs.

2010 Mathematics Subject Classification: 13D30; 18E05; 18E30; 18E10.

\section{Introduction}

The recollement of triangulated categories was introduced in a geometric setting by Beilinson, Bernstein and Deligne [BBD]. Nowadays it has become very powerful in understanding relationships among three algebraic, geometric or topological objects. A fundamental example of the recollement of abelian categories is due to MacPherson and Vilonen [MV], in which it first appeared as an inductive step in the construction of perverse sheaves. Later, Wang and Lin [WL] defined the notion of recollement of addtive categories, which unifies the recollment of abelian categories and the recollement of triangulated categories. Recently, Chen, Liu and Yang [CLY] introduced localization sequences and colocalization sequences of additive categories which are similar to lower recollements and upper recollements of triangulated categories respectively. They consider recollements of additive categories by cotorsion pairs in triangulated categories. Based on this fact that a co-t-structure is a special cotorsion pair, they give some recollements of additive categories associated to concentric twin cotorsion pairs in a triangulated category by doing quotient.

Extriangulated categories were recently introduced by Nakaoka and Palu [NP] by extracting those properties of $\mathrm{Ext}^{1}$ on exact categories (which is itself a generalisation of the concept of a module category and an abelian category) and on triangulated categories that seem relevant

\footnotetext{
^ Yonggang Hu was supported by the China Scholarship Council (CSC) and Panyue Zhou was supported by the National Natural Science Foundation of China (Grant No. 11901190 and 11671221), and by the Hunan Provincial Natural Science Foundation of China (Grant No. 2018JJ3205), and by the Scientific Research Fund of Hunan Provincial Education Department (Grant No. 19B239).
} 
from the point of view of cotorsion pairs. In particular, exact categories and triangulated categories are extriangulated categories. There are a lot of examples of extriangulated categories which are neither exact categories nor triangulated categories, see [NP, ZZ]. Hence, many results hold on exact categories and triangulated categories can be unified in the same framework. Based on this idea, we extend Chen-Liu-Yang's results to extriangulated categories.

Our main result is the following.

Theorem 1.1. (See Theorem 3.8 for more details) Let $\mathcal{C}$ be an extriangulated category with enough projectives and enough injectives, and $(\mathcal{S}, \mathcal{T}),(\mathcal{U}, \mathcal{V})$ and $(\mathcal{X}, \mathcal{Y})$ be cotorsion pairs on $\mathcal{C}$ with $\mathcal{W}=\mathcal{S} \cap \mathcal{T}=\mathcal{U} \cap \mathcal{V}=\mathcal{X} \cap \mathcal{Y}$. If $\mathcal{X} \cap \mathcal{T}=\mathcal{V}, \mathcal{Y} \subseteq \mathcal{T}, \Sigma \mathcal{V} \subseteq \mathcal{V}$ and $\Sigma \mathcal{Y} \subseteq \mathcal{Y}$, then there are two recollements of additive categories as follows:
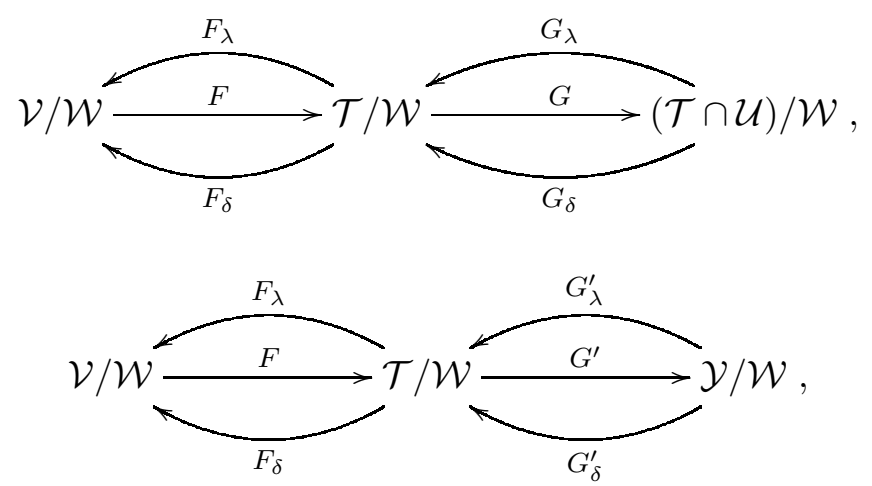

where $F, G_{\lambda}$ and $G_{\delta}$ are the full embeddings.

Our main result generalizes Chen-Liu-Yang's results on a triangulated category and is new for an exact category case. In particular, applying our main results to complex categories, we reobtain the Krause's recollement. In fact, without considering homotopy categories, we reprove the existence of Krause's recollement by our main resluts.

This article is organised as follows: In Section 2, we review some basic concepts and results concerning extriangulated categories. In Section 3, we show our main results. In Section 4, we give some applications to illustrate our main results.

\section{Preliminaries}

Let us briefly recall some definitions and basic properties of extriangulated categories from [NP. We omit some details here, but the reader can find them in $[\mathrm{NP}$.

Let $\mathcal{C}$ be an additive category equipped with an additive bifunctor

$$
\mathbb{E}: \mathcal{C}^{\mathrm{op}} \times \mathcal{C} \rightarrow \mathrm{Ab}
$$

where $\mathrm{Ab}$ is the category of abelian groups. For any objects $A, C \in \mathcal{C}$, an element $\delta \in \mathbb{E}(C, A)$ is called an $\mathbb{E}$-extension. Let $\mathfrak{s}$ be a correspondence which associates an equivalence class

$$
\mathfrak{s}(\delta)=[A \stackrel{x}{\longrightarrow} B \stackrel{y}{\longrightarrow} C]
$$


to any $\mathbb{E}$-extension $\delta \in \mathbb{E}(C, A)$. This $\mathfrak{s}$ is called a realization of $\mathbb{E}$, if it makes the diagrams in $[\mathrm{NP}$, Definition 2.9] commutative. A triplet $(\mathcal{C}, \mathbb{E}, \mathfrak{s})$ is called an extriangulated category if it satisfies the following conditions.

(1) $\mathbb{E}: \mathcal{C}^{\mathrm{op}} \times \mathcal{C} \rightarrow \mathrm{Ab}$ is an additive bifunctor.

(2) $\mathfrak{s}$ is an additive realization of $\mathbb{E}$.

(3) $\mathbb{E}$ and $\mathfrak{s}$ satisfy the compatibility conditions in [NP, Definition 2.12].

Remark 2.1. Note that both exact categories and triangulated categories are extriangulated categories, see [NP, Example 2.13] and extension closed subcategories of triangulated categories are again extriangulated, see [NP, Remark 2.18]. Moreover, there exist extriangulated categories which are neither exact categories nor triangulated categories, see $[\mathrm{NP}$, Proposition 3.30] and [ZZ, Example 4.14].

We will use the following terminology.

Definition 2.2. $[\mathrm{NP}]$ Let $(\mathcal{C}, \mathbb{E}, \mathfrak{s})$ be an extriangulated category.

(1) A sequence $A \stackrel{x}{\longrightarrow} B \stackrel{y}{\longrightarrow} C$ is called a conflation if it realizes some $\mathbb{E}$-extension $\delta \in$ $\mathbb{E}(C, A)$. In this case, $x$ is called an inflation and $y$ is called a deflation.

(2) If a conflation $A \stackrel{x}{\longrightarrow} B \stackrel{y}{\longrightarrow} C$ realizes $\delta \in \mathbb{E}(C, A)$, we call the pair $(A \stackrel{x}{\longrightarrow} B \stackrel{y}{\longrightarrow} C, \delta)$ an $\mathbb{E}$-triangle, and write it in the following way.

$$
A \stackrel{x}{\longrightarrow} B \stackrel{y}{\longrightarrow} C \stackrel{\delta}{\rightarrow-}
$$

We usually do not write this " $\delta$ " if it is not used in the argument.

(3) Let $A \stackrel{x}{\longrightarrow} B \stackrel{y}{\longrightarrow} C \stackrel{-\delta}{\rightarrow}$ and $A^{\prime} \stackrel{x^{\prime}}{\longrightarrow} B^{\prime} \stackrel{y^{\prime}}{\longrightarrow} C^{\prime} \stackrel{\delta^{\prime}}{\rightarrow}$ be any pair of $\mathbb{E}$-triangles. If a triplet $(a, b, c)$ realizes $(a, c): \delta \rightarrow \delta^{\prime}$, then we write it as

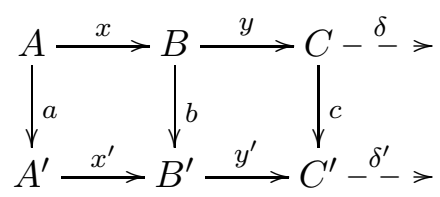

and call $(a, b, c)$ a morphism of $\mathbb{E}$-triangles.

(4) An object $P \in \mathcal{C}$ is called projective if for any $\mathbb{E}$-triangle $A \stackrel{x}{\longrightarrow} B \stackrel{y}{\longrightarrow} C \stackrel{\delta}{\rightarrow}$ and any morphism $c \in \mathcal{C}(P, C)$, there exists $b \in \mathcal{C}(P, B)$ satisfying $y b=c$. We denote the subcategory of projective objects by $\mathcal{P} \subseteq \mathcal{C}$. Dually, the subcategory of injective objects is denoted by $\mathcal{I} \subseteq \mathcal{C}$.

(5) We say that $\mathcal{C}$ has enough projective objects if for any object $C \in \mathcal{C}$, there exists an $\mathbb{E}$-triangle $A \stackrel{x}{\longrightarrow} P \stackrel{y}{\longrightarrow} C \stackrel{\delta}{\rightarrow}$ satisfying $P \in \mathcal{P}$. We can define the notion of having enough injectives dually. 
Assume that $(\mathcal{C}, \mathbb{E}, \mathfrak{s})$ is an extriangulated category. By Yoneda's lemma, any $\mathbb{E}$-extension $\delta \in \mathbb{E}(C, A)$ induces natural transformations

$$
\delta_{\sharp}: \mathcal{C}(-, C) \Rightarrow \mathbb{E}(-, A) \text { and } \delta^{\sharp}: \mathcal{C}(A,-) \Rightarrow \mathbb{E}(C,-) .
$$

For any $X \in \mathcal{C}$, these $\left(\delta_{\sharp}\right)_{X}$ and $\delta_{X}^{\sharp}$ are given as follows:

(1) $\left(\delta_{\sharp}\right)_{X}: \mathcal{C}(X, C) \rightarrow \mathbb{E}(X, A) ; f \mapsto f^{*} \delta$.

(2) $\delta_{X}^{\sharp}: \mathcal{C}(A, X) \rightarrow \mathbb{E}(C, X) ; g \mapsto g_{*} \delta$.

Lemma 2.3. Let $(\mathcal{C}, \mathbb{E}, \mathfrak{s})$ be an extriangulated category, and

$$
A \stackrel{x}{\longrightarrow} B \stackrel{y}{\longrightarrow} C-\stackrel{\delta}{\rightarrow}
$$

an $\mathbb{E}$-triangle. Then we have the following long exact sequence:

$$
\begin{aligned}
& \mathcal{C}(-, A) \stackrel{\mathcal{C}(-, x)}{\longrightarrow} \mathcal{C}(-, B) \stackrel{\mathcal{C}(-, y)}{\longrightarrow} \mathcal{C}(-, C) \stackrel{\delta_{-}^{\sharp}}{\longrightarrow} \mathbb{E}(-, A) \stackrel{\mathbb{E}(-, x)}{\longrightarrow} \mathbb{E}(-, B) \stackrel{\mathbb{E}(-, y)}{\longrightarrow} \mathbb{E}(-, C) ; \\
& \mathcal{C}(C,-) \stackrel{\mathcal{C}(y,-)}{\longrightarrow} \mathcal{C}(B,-) \stackrel{\mathcal{C}(x,-)}{\longrightarrow} \mathcal{C}(A,-) \stackrel{\delta_{\sharp}^{-}}{\longrightarrow} \mathbb{E}(C,-) \stackrel{\mathbb{E}(y,-)}{\longrightarrow} \mathbb{E}(B,-) \stackrel{\mathbb{E}(x,-)}{\longrightarrow} \mathbb{E}(A,-) .
\end{aligned}
$$

Proof. This follows from Proposition 3.3 and Proposition 3.11 in [NP].

Now we recall higher extension groups from [LN].

Assume that $(\mathcal{C}, \mathbb{E}, \mathfrak{s})$ is an extriangulated category with enough projectives and enough injectives. For two subcategories $\mathcal{X}, \mathcal{Y}$ of $\mathcal{C}$, Cone $(\mathcal{X}, \mathcal{Y})$ is defined to be the subcategory of $\mathcal{C}$, consisting of objects $C$ which admits an $\mathbb{E}$-triangle $X \stackrel{x}{\longrightarrow} Y \stackrel{y}{\longrightarrow} C-\stackrel{\delta}{\longrightarrow}$ where $X \in \mathcal{X}$ and $Y \in \mathcal{Y}$. Dually we can define $\operatorname{CoCone}(\mathcal{X}, \mathcal{Y})$.

For a subcategory $\mathcal{C}_{1} \subseteq \mathcal{C}$, put $\Omega^{0} \mathcal{C}_{1}=\mathcal{C}_{1}$, and define $\Omega^{i} \mathcal{C}_{1}$ for $i>0$ inductively by

$$
\Omega^{i} \mathcal{C}_{1}=\Omega\left(\Omega^{i-1} \mathcal{C}_{1}\right)=\operatorname{CoCone}\left(\mathcal{P}, \Omega^{i-1} \mathcal{C}_{1}\right)
$$

We call $\Omega^{i} \mathcal{C}_{1}$ the $i$-th syzygy of $\mathcal{C}_{1}$. Dually we define the $i$-th cosyzygy $\Sigma^{i} \mathcal{C}_{1}$ by $\Sigma^{0} \mathcal{C}_{1}=\mathcal{C}_{1}$ and $\Sigma^{i} \mathcal{C}_{1}=\operatorname{Cone}\left(\Sigma^{i-1} \mathcal{C}_{1}, \mathcal{I}\right)$ for $i>0$.

Let $X$ be any object in $\mathcal{C}$. It admits an $\mathbb{E}$-triangle

$$
X \rightarrow I^{0} \rightarrow \Sigma X \rightarrow \quad\left(\text { resp. } \Omega X \rightarrow P_{0} \rightarrow X-\rightarrow\right)
$$

where $I^{0} \in \mathcal{I}$ (resp. $\left.P_{0} \in \mathcal{P}\right)$. We can obtain $\mathbb{E}$-triangles

$$
\Sigma^{i} X \rightarrow I^{i} \rightarrow \Sigma^{i+1} X-\rightarrow\left(\operatorname{resp} . \Omega^{i+1} \rightarrow P_{i} \rightarrow \Omega^{i} X-\rightarrow\right),
$$

for $i>0$ recursively.

Liu and Nakaoka [LN] defined higher extension groups as

$$
\mathbb{E}^{i+1}(X, Y):=\mathbb{E}\left(X, \Sigma^{i} Y\right) \cong \mathbb{E}\left(\Omega^{i} X, Y\right)
$$

for $i \geq 0$, and proved the following. 
Lemma 2.4. [LN, Proposition 5.2] Let $(\mathcal{C}, \mathbb{E}, \mathfrak{s})$ be an extriangulated category with enough projectives and enough injectives, and

$$
A \stackrel{x}{\longrightarrow} B \stackrel{y}{\longrightarrow} C-\stackrel{\delta}{-}>
$$

an $\mathbb{E}$-triangle. Then we have the following long exact sequence:

$$
\begin{aligned}
& \cdots \rightarrow \mathbb{E}^{i}(-, A) \rightarrow \mathbb{E}^{i}(-, B) \rightarrow \mathbb{E}^{i}(-, C) \rightarrow \mathbb{E}^{i+1}(-, A) \rightarrow \mathbb{E}^{i+1}(-, B) \rightarrow \mathbb{E}^{i+1}(-, C) \rightarrow \cdots(i \geq 1) ; \\
& \cdots \rightarrow \mathbb{E}^{i}(C,-) \rightarrow \mathbb{E}^{i}(B,-) \rightarrow \mathbb{E}^{i}(A,-) \rightarrow \mathbb{E}^{i+1}(C,-) \rightarrow \mathbb{E}^{i+1}(B,-) \rightarrow \mathbb{E}^{i+1}(A,-) \rightarrow \cdots(i \geq 1) .
\end{aligned}
$$

Remark 2.5. [ZhZ, Lemma 2.14] Let $(\mathcal{C}, \mathbb{E}, \mathfrak{s})$ be an extriangulated category with enough projectives and enough injectives. Then

(a) $P$ is projective object in $\mathcal{C}$ if and only if $\mathbb{E}^{i}(P, C)=0$, for any $C \in \mathcal{C}$ and $i \geq 1$.

(b) $I$ is injective object in $\mathcal{C}$ if and only if $\mathbb{E}^{i}(C, I)=0$, for any $C \in \mathcal{C}$ and $i \geq 1$.

Let $\mathcal{C}$ be an additive category. For two objects $A, B$ in $\mathcal{X}$ denote by $\mathcal{X}(A, B)$ the subgroup of $\operatorname{Hom}_{\mathcal{C}}(A, B)$ consisting of those morphisms which factor through an object in $\mathcal{X}$. Denote by $\mathcal{C} / \mathcal{X}$ the quotient category of $\mathcal{C}$ modulo $\mathcal{X}$ : the objects are the same as the ones in $\mathcal{C}$, for two objects $A$ and $B$ the Hom space is given by the quotient group $\operatorname{Hom}_{\mathcal{C}}(A, B) / \mathcal{X}(A, B)$. Note that the quotient category $\mathcal{C} / \mathcal{X}$ is an additive category. We denote $\bar{f}$ the image of $f: A \rightarrow B$ of $\mathcal{C}$ in $\mathcal{C} / \mathcal{X}$.

Remark 2.6. If $\mathcal{X}$ is closed under direct summands, for any $C \in \mathcal{C}$ we have $C \cong 0$ in $\mathcal{C} / \mathcal{X}$ if and only if $C \in \mathcal{X}$.

Definition 2.7. $[\mathrm{NP}$, Definition 4.1] Assume that $(\mathcal{C}, \mathbb{E}, \mathfrak{s})$ is an extriangulated category. Let $\mathcal{U}$ and $\mathcal{V}$ be two subcategories of $\mathcal{C}$. We call $(\mathcal{U}, \mathcal{V})$ a cotorsion pair if it satisfies the following conditions:

(a) $\mathbb{E}(\mathcal{U}, \mathcal{V})=0$.

(b) For any object $C \in \mathcal{C}$, there are two $\mathbb{E}$-triangles

$$
V_{C} \rightarrow U_{C} \rightarrow C-\rightarrow \text { and } C \rightarrow V^{C} \rightarrow U^{C} \rightarrow
$$

satisfying $U_{C}, U^{C} \in \mathcal{U}$ and $V_{C}, V^{C} \in \mathcal{V}$.

By definition of a cotorsion pair, we can immediately conclude:

Remark 2.8. Assume that $(\mathcal{C}, \mathbb{E}, \mathfrak{s})$ is an extriangulated category. Let $(\mathcal{U}, \mathcal{V})$ be a cotorsion pair on $\mathcal{C}$. Then

(a) $C$ belongs to $\mathcal{U}$ if and only if $\mathbb{E}(C, V)=0$;

(b) $C$ belongs to $\mathcal{V}$ if and only if $\mathbb{E}(\mathcal{U}, C)=0$;

(c) $\mathcal{U}$ and $\mathcal{V}$ are closed under direct sums, direct summands and extensions.

Definition 2.9. [NP, Definition 4.12] Assume that $(\mathcal{C}, \mathbb{E}, \mathfrak{s})$ is an extriangulated category. Let $(\mathcal{S}, \mathcal{T})$ and $(\mathcal{U}, \mathcal{V})$ be cotorsion pairs on $\mathcal{C}$. Then the pair $T C P:=((\mathcal{S}, \mathcal{T}),(\mathcal{U}, \mathcal{V}))$ is called a 
twin cotorsion pair if it satisfies $\mathbb{E}(\mathcal{S}, \mathcal{V})=0$. Note that this condition is equivalent to $\mathcal{S} \subseteq \mathcal{U}$, and also to $\mathcal{V} \subseteq \mathcal{T}$. If it moreover it satisfies $\mathcal{S} \cap \mathcal{T}=\mathcal{U} \cap \mathcal{V}$, then $T C P$ is called a concentric twin cotorsion pair.

Lemma 2.10. Assume that $(\mathcal{C}, \mathbb{E}, \mathfrak{s})$ is an extriangulated category. Let $(\mathcal{U}, \mathcal{V})$ be a cotorsion pair on $\mathcal{C}$ and $\mathcal{W}:=\mathcal{U} \cap \mathcal{V}$. Then $(\mathcal{C} / \mathcal{W})(\mathcal{U} / \mathcal{W}, \mathcal{V} / \mathcal{W})=0$

Proof. Let $f \in \mathcal{C}(U, V)$ be any morphism, where $U \in \mathcal{U}$ and $V \in \mathcal{V}$. By definition of a cotorsion pair, there exists an $\mathbb{E}$-triangle

$$
V^{\prime} \stackrel{x}{\longrightarrow} U^{\prime} \stackrel{y}{\longrightarrow} V_{-\stackrel{\delta}{\rightarrow}}^{\rightarrow}
$$

where $V^{\prime} \in \mathcal{V}$ and $U^{\prime} \in \mathcal{U}$. Since $\mathcal{V}$ is closed under extensions, we have $U^{\prime} \in \mathcal{V}$ and then $U^{\prime} \in \mathcal{U} \cap \mathcal{V}=\mathcal{W}$. Applying the functor $\mathcal{C}(U,-)$ to the $\mathbb{E}$-triangle $V^{\prime} \stackrel{x}{\longrightarrow} U^{\prime} \stackrel{y}{\longrightarrow} V_{--}^{-}$, by Lemma 2.3, we have the following exact sequence:

$$
\mathcal{C}\left(U, U^{\prime}\right) \stackrel{\mathcal{C}(U, y)}{\longrightarrow} \mathcal{C}(U, V) \rightarrow \mathbb{E}\left(U, V^{\prime}\right)=0 .
$$

It follows that there exists a morphism $a: U \rightarrow U^{\prime}$ such that $f=y \circ a$. Since $U^{\prime} \in \mathcal{W}$, this means $\bar{f}=0$.

Lemma 2.11. Assume that $(\mathcal{C}, \mathbb{E}, \mathfrak{s})$ is an extriangulated category. Let $(\mathcal{U}, \mathcal{V})$ be a cotorsion pair on $\mathcal{C}$ and $\mathcal{W}:=\mathcal{U} \cap \mathcal{V}$, and let $f: A \rightarrow B$ be any morphism in $\mathcal{C}$.

(1) Let

$$
\begin{aligned}
& V_{A} \rightarrow U_{A} \stackrel{u_{A}}{\longrightarrow} A-\rightarrow \\
& V_{B} \rightarrow U_{B} \stackrel{u_{B}}{\longrightarrow} B-\rightarrow
\end{aligned}
$$

be any $\mathbb{E}$-triangles satisfying $U_{A}, U_{B} \in \mathcal{U}$ and $V_{A}, V_{B} \in \mathcal{V}$. Then there exists a morphism $f_{U} \in \mathcal{C}\left(U_{A}, U_{B}\right)$ such that

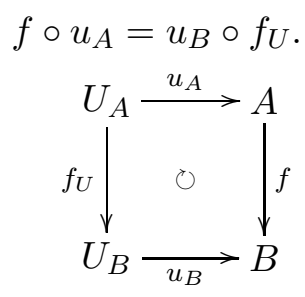

Moreover, $f_{U}$ with this property is unique in $(\mathcal{C} / \mathcal{W})\left(U_{A}, U_{B}\right)$.

(2) Dually, for any $\mathbb{E}$-triangles

$$
\begin{aligned}
& A \rightarrow V_{A}^{\prime} \rightarrow U_{A}^{\prime}-\rightarrow \\
& B \rightarrow V_{B}^{\prime} \rightarrow U_{B}^{\prime}-\rightarrow
\end{aligned}
$$

with $U_{A}^{\prime}, U_{B}^{\prime} \in \mathcal{U}$ and $V_{A}^{\prime}, V_{B}^{\prime} \in \mathcal{V}$, there exists a morphism $f_{V}^{\prime} \in \mathcal{C}\left(V_{A}^{\prime}, V_{B}^{\prime}\right)$ compatible with $f$, uniquely up to $\mathcal{W}$. 
Proof. We only show (1). Existence immediately follows from $\mathbb{E}\left(U_{A}, V_{B}\right)=0$. Moreover if $f_{U}^{1}$ and $f_{U}^{2}$ in $\mathcal{C}\left(U_{A}, U_{B}\right)$ satisfies

$$
f_{U}^{1} \circ u_{A}=u_{B} \circ f_{U}=f_{U}^{2} \circ u_{A}
$$

then by $\left(f_{U}^{1}-f_{U}^{2}\right) \circ u_{A}=0$, there exists $w \in \mathcal{C}\left(U_{A}, V_{B}\right)$ such that $f_{U}^{1}-f_{U}^{2}$ factors through $w$.

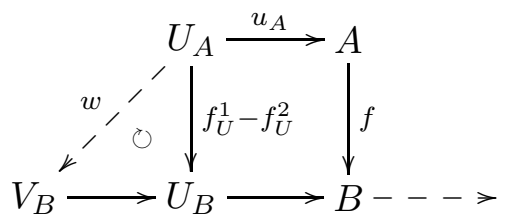

By Lemma 2.10 we have $\bar{w}=0$, and then $\overline{f_{U}^{1}}=\overline{f_{U}^{2}}$.

Lemma 2.12. Assume that $(\mathcal{C}, \mathbb{E}, \mathfrak{s})$ is an extriangulated category. Let $(\mathcal{U}, \mathcal{V})$ be a cotorsion pair on $\mathcal{C}, \mathcal{W}:=\mathcal{U} \cap \mathcal{V}$ and $C$ be any object in $\mathcal{C}$.

(1) For any $\mathbb{E}$-triangles

$$
\begin{gathered}
V \rightarrow U \stackrel{u}{\longrightarrow} C-\rightarrow \\
V^{\prime} \rightarrow U^{\prime} \stackrel{u^{\prime}}{\longrightarrow} C-\rightarrow
\end{gathered}
$$

satisfying $U, U^{\prime} \in \mathcal{U}$ and $V, V^{\prime} \in \mathcal{V}$, there exists a morphism $s \in \mathcal{C}\left(U, U^{\prime}\right)$ compatible with $u$ and $u^{\prime}$, such that $\bar{s}$ is an isomorphism.

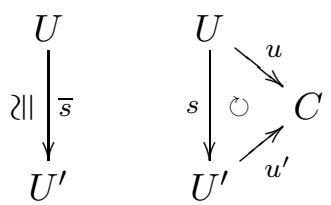

(2) Dually, those $V$ appearing in $\mathbb{E}$-triangles

$$
C \rightarrow V \rightarrow U \rightarrow \quad \text { where } U \in \mathcal{U}, V \in \mathcal{V}
$$

are isomorphic in $\mathcal{C} / \mathcal{W}$.

Proof. This immediately follows from Lemma 2.11.

\section{Recollements of additive categories}

In this section, we will prove our main results, and need to do some preparations as follows.

Definition 3.1. WL, Definition 2.1] and [CLY, Definition 3.1] Let $\mathcal{A}^{\prime} \stackrel{F}{\longrightarrow} \mathcal{A} \stackrel{G}{\longrightarrow} \mathcal{A}^{\prime \prime}$ be a sequence of additive functors between additive categories. We say it is a localization sequence if the following conditions hold:

(L1) The functor $F$ is fully faithful and has a right adjoint $F_{\delta}$. 
(L2) The functor $G$ has a fully faithful right adjoint $G_{\delta}$.

(L3) There exists an equality of additive subcategories $\operatorname{Im} F=\operatorname{Ker} G$, where $\operatorname{Im} F=\left\{A \in \mathcal{A} \mid A \cong F(X)\right.$ for some $\left.X \in \mathcal{A}^{\prime}\right\}$ and $\operatorname{Ker} G=\left\{A \in \mathcal{A} \mid G(A)=0\right.$ in $\left.\mathcal{A}^{\prime \prime}\right\}$.

Colocalization sequence of additive categories is defined dually. A sequence of additive categories $\mathcal{A}^{\prime} \stackrel{F}{\longrightarrow} \mathcal{A} \stackrel{G}{\longrightarrow} \mathcal{A}^{\prime \prime}$ is called a recollement if it is both a localization sequence and a colocalization sequence.

From now on, we assume that $(\mathcal{C}, \mathbb{E}, \mathfrak{s})$ is an extriangulated category with enough projectives and enough injectives.

Definition 3.2. [B, Definition 3.1.1] Let $\mathcal{A}$ and $\mathcal{B}$ be additive categories and $F: \mathcal{A} \rightarrow \mathcal{B}$ a functor. For any $B \in \mathcal{B}$, a reflection of $B$ along $F$ is a pair $\left(Q_{B}, \eta_{B}\right)$ of $Q_{B} \in \mathcal{A}$ and $\eta_{B} \in \mathcal{B}\left(B, F\left(Q_{B}\right)\right)$, satisfying the universality that for any $A \in \mathcal{A}$ and any $b \in \mathcal{B}(B, F(A))$, there exists a unique morphism $a \in \mathcal{A}\left(Q_{B}, A\right)$ such that $F(a) \circ \eta_{B}=b$.

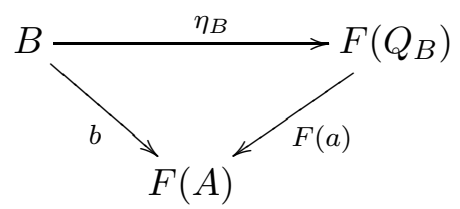

A coreflection is defined dually.

Lemma 3.3. Let $((\mathcal{S}, \mathcal{T}),(\mathcal{U}, \mathcal{V}))$ be a concentric twin cotorsion pair on $\mathcal{C}$ with $\mathcal{W}=\mathcal{S} \cap \mathcal{T}$. Then the inclusion $\mathcal{V} / \mathcal{W} \hookrightarrow \mathcal{T} / \mathcal{W}$ admits an additive left adjoint $F_{\lambda}: \mathcal{T} / \mathcal{W} \rightarrow \mathcal{V} / \mathcal{W}$. Indeed, for any $T \in \mathcal{T}$ and $\mathbb{E}$-triangle

$$
T \stackrel{v_{T}}{\longrightarrow} V_{T} \longrightarrow U_{T}-\rightarrow
$$

with $U_{T} \in \mathcal{U}$ and $V_{T} \in \mathcal{V}, \overline{v_{T}}: T \rightarrow V_{T}$ gives a reflection of $T$ along the inclusion $\mathcal{V} / \mathcal{W} \hookrightarrow \mathcal{T} / \mathcal{W}$, where $F_{\lambda}(T)=V_{T}$.

Proof. It is enough to prove that for any $T \in \mathcal{T}, V \in \mathcal{V}$ and $f \in \mathcal{C}(T, V)$, there exists a morphism $g \in \mathcal{C}\left(V_{T}, V\right)$ such that $f=g \circ v_{T}$, uniquely in $(\mathcal{C} / \mathcal{W})\left(V_{T}, V\right)$.

Applying the functor $\mathcal{C}(-, V)$ to the $\mathbb{E}$-triangle $T \stackrel{v_{T}}{\longrightarrow} V_{T} \longrightarrow U_{T} \rightarrow$, we have the following exact sequence:

$$
\mathcal{C}\left(V_{T}, V\right) \stackrel{\mathcal{C}\left(v_{T}, V\right)}{\longrightarrow} \mathcal{C}(T, V) \rightarrow \mathbb{E}\left(U_{T}, V\right)=0 .
$$

Thus there exists a morphism $g \in \mathcal{C}\left(V_{T}, V\right)$ such that $f=g \circ v_{T}$ and then $\bar{f}=\bar{g} \circ \overline{v_{T}}$.

To prove uniqueness now, suppose that there exists a morphism $g \in \mathcal{C}\left(V_{T}, V\right)$ such that $\bar{f}=\overline{g^{\prime}} \circ \overline{v_{T}}$. Then $\left(\bar{g}-\overline{g^{\prime}}\right) \circ v_{T}=0$, that is, $\left(g-g^{\prime}\right) \circ v_{T}$ factors through some $W_{0} \in \mathcal{W}$. Let $\left(g-g^{\prime}\right) \circ v_{T}=b \circ a$ with $a: T \rightarrow W_{0}$ and $b: W_{0} \rightarrow V$.

Applying the functor $\mathcal{C}\left(-, W_{0}\right)$ to the $\mathbb{E}$-triangle $T \stackrel{v_{T}}{\longrightarrow} V_{T} \longrightarrow U_{T}-\rightarrow$, we have the following exact sequence:

$$
\mathcal{C}\left(V_{T}, W_{0}\right) \stackrel{\mathcal{C}\left(v_{T}, W_{0}\right)}{\longrightarrow} \mathcal{C}\left(T, W_{0}\right) \rightarrow \mathbb{E}\left(U_{T}, W_{0}\right)=0 .
$$


Thus there exists a morphism $c \in \mathcal{C}\left(V_{T}, W_{0}\right)$ such that $a=c \circ v_{T}$ and then $\left(g-g^{\prime}-b \circ c\right) \circ v_{T}=0$. By Lemma 2.3, there exists a morphism $d \in \mathcal{C}\left(U_{T}, V\right)$ such that $\left(g-g^{\prime}\right)-b \circ c=d \circ e$. It follows that $\bar{g}-\overline{g^{\prime}}=\bar{d} \circ \bar{e}$. By Lemma 2.10, we have $(\mathcal{C} / \mathcal{W})\left(U_{T}, V\right)=0$ implies $\bar{g}=\overline{g^{\prime}}$. Therefore, $v_{T}: T \rightarrow V_{T}$ gives a reflection of $T$ along the inclusion $\mathcal{V} / \mathcal{W} \hookrightarrow \mathcal{T} / \mathcal{W}$. Namely, $F_{\lambda}: \mathcal{T} / \mathcal{W} \rightarrow \mathcal{V} / \mathcal{W}$ is left adjoint to the inclusion $\mathcal{V} / \mathcal{W} \rightarrow \mathcal{T} / \mathcal{W}$

Lemma 3.4. Let $((\mathcal{S}, \mathcal{T}),(\mathcal{U}, \mathcal{V}))$ be a concentric twin cotorsion pair on $\mathcal{C}$ with $\mathcal{W}=\mathcal{S} \cap \mathcal{T}$. If $\Sigma \mathcal{V} \subseteq \mathcal{V}$, then there exists the following colocalization sequence of additive categories:

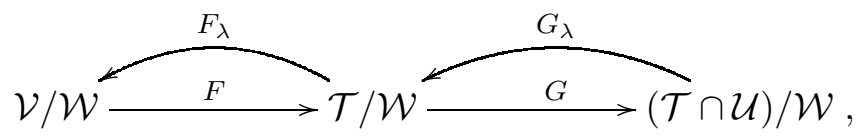

where $F$ and $G_{\lambda}$ are the full embeddings.

Proof. Assume that $F: \mathcal{V} / \mathcal{W} \rightarrow \mathcal{T} / \mathcal{W}$ is the full embedding. We define $G: \mathcal{T} / \mathcal{W} \rightarrow(\mathcal{T} \cap \mathcal{U}) / \mathcal{W}$ in the following way. For any $T \in \mathcal{T}, G(T)=Z_{T}$ appearing in an $\mathbb{E}$-triangle $V_{T} \longrightarrow Z_{T} \longrightarrow$ $T \rightarrow$ with $Z_{T} \in \mathcal{T} \cap \mathcal{U}$ and $V_{T} \in \mathcal{V}$. By [ZW, Definition 3.13], we know that $G$ is an additive functor and a right adjoint of the inclusion $(\mathcal{T} \cap \mathcal{U}) / \mathcal{W} \rightarrow \mathcal{T} / \mathcal{W}$, denoted by $G_{\lambda}$. We claim that $\operatorname{Im} F=\operatorname{Ker} G$. It is easy to see that $\operatorname{Im} F=\mathcal{V}$ since $F$ is the full embedding. For any $V \in \mathcal{V}$, there exists an $\mathbb{E}$-triangle

$$
V_{0} \longrightarrow W_{0} \longrightarrow V-\rightarrow
$$

with $W_{0} \in \mathcal{W}$ and $V_{0} \in \mathcal{V}$. Hence, $G(V)=W_{0}$ in $(\mathcal{T} \cap \mathcal{U}) / \mathcal{W}$. So we know that $V \in \operatorname{Ker} G$ by Remark 2.6. Then $\operatorname{Im} F \subseteq \operatorname{Ker} G$. On the other hand, for any $T \in \operatorname{Ker} G$, there exists an $\mathbb{E}$-triangle

$$
V_{T} \longrightarrow Z_{T} \longrightarrow T--\rightarrow
$$

with $Z_{T} \in \mathcal{W}$ and $V_{T} \in \mathcal{V}$. Since $\Sigma \mathcal{V} \subseteq \mathcal{V}$, we have $\mathbb{E}\left(U, \Sigma V_{T}\right)=0$. Applying the functor $\mathcal{C}(\mathcal{U},-)$ to the $\mathbb{E}$-triangle $T \stackrel{v_{T}}{\longrightarrow} V_{T} \longrightarrow U_{T}-\rightarrow$, by Lemma 2.5, we obtain the following exact sequence:

$$
\mathbb{E}\left(\mathcal{U}, Z_{T}\right)=0 \rightarrow \mathbb{E}(\mathcal{U}, T) \rightarrow \mathbb{E}\left(\mathcal{U}, \Sigma V_{T}\right)=0 .
$$

It follows that $\mathbb{E}(\mathcal{U}, T)=0$ and then $T \in \mathcal{V}$. Thus $\operatorname{Im} F=\operatorname{Ker} G$. Let $F_{\lambda}$ be as in Lemma 3.3. Then $\left(F_{\lambda}, F\right)$ is an adjoint pair. This completes the proof.

Lemma 3.5. Let $(\mathcal{U}, \mathcal{V})$ be a cotorsion pair and $((\mathcal{S}, \mathcal{T}),(\mathcal{X}, \mathcal{Y}))$ a concentric twin cotorsion pair on $\mathcal{C}$ with $\mathcal{W}=\mathcal{S} \cap \mathcal{T}$. If $\mathcal{T} \cap \mathcal{X}=\mathcal{V}$, then the inclusion $\mathcal{V} / \mathcal{W} \rightarrow \mathcal{T} / \mathcal{W}$ admits an additive right adjoint functor $F_{\delta}: \mathcal{T} / \mathcal{W} \rightarrow \mathcal{V} / \mathcal{W}$. Indeed, for any $T \in \mathcal{T}$ and $\mathbb{E}$-triangle

$$
Y_{T} \longrightarrow V_{T} \stackrel{x_{T}}{\longrightarrow} T \rightarrow
$$

with $Y_{T} \in \mathcal{Y}$ and $V_{T} \in \mathcal{V}, \overline{x_{T}}: V_{T} \rightarrow T$ gives a coreflection of $T$ along the inclusion $\mathcal{V} / \mathcal{W} \hookrightarrow$ $\mathcal{T} / \mathcal{W}$, where $F_{\delta}(T)=V_{T}$. 
Proof. For any $T \in \mathcal{T}$, there exists an $\mathbb{E}$-triangle

$$
Y_{T} \longrightarrow V_{T} \stackrel{x_{T}}{\longrightarrow} T-\rightarrow
$$

with $Y_{T} \in \mathcal{Y}$ and $V_{T} \in \mathcal{X}$. Since $\mathcal{Y} \subseteq \mathcal{T}$ and $\mathcal{T}$ is closed under extensions, we have $V_{T} \in \mathcal{T}$. Thus $V_{T} \in \mathcal{X} \cap \mathcal{T}=\mathcal{V}$. The remaining proof is similar to Lemma 3.3

Lemma 3.6. Let $((\mathcal{S}, \mathcal{T}),(\mathcal{X}, \mathcal{Y}))$ a concentric twin cotorsion pair on $\mathcal{C}$ with $\mathcal{W}=\mathcal{S} \cap \mathcal{T}$. Then the inclusion $\mathcal{Y} / \mathcal{W} \hookrightarrow \mathcal{T} / \mathcal{W}$ admits an additive left adjoint $G^{\prime}: \mathcal{T} / \mathcal{W} \rightarrow \mathcal{Y} / \mathcal{W}$. Indeed, for any $T \in \mathcal{T}$ and $\mathbb{E}$-triangle

$$
T \stackrel{y_{T}}{\longrightarrow} Y_{T} \longrightarrow X_{T}-\rightarrow
$$

with $Y_{T} \in \mathcal{Y}$ and $X_{T} \in \mathcal{X}, \overline{y_{T}}: T \rightarrow Y_{T}$ gives a reflection of $T$ along the inclusion $\mathcal{Y} / \mathcal{W} \hookrightarrow$ $\mathcal{T} / \mathcal{W}$, where $G^{\prime}(T)=Y_{T}$.

Proof. The proof is similar to Lemma 3.3.

Lemma 3.7. Let $(\mathcal{U}, \mathcal{V})$ be a cotorsion pair and $((\mathcal{S}, \mathcal{T}),(\mathcal{X}, \mathcal{Y}))$ a concentric twin cotorsion pair on $\mathcal{C}$ with $\mathcal{W}=\mathcal{S} \cap \mathcal{T}$. If $\mathcal{T} \cap \mathcal{X}=\mathcal{V}$ and $\Sigma \mathcal{Y} \subseteq \mathcal{Y}$, then there exists the following localization sequence of additive categories:

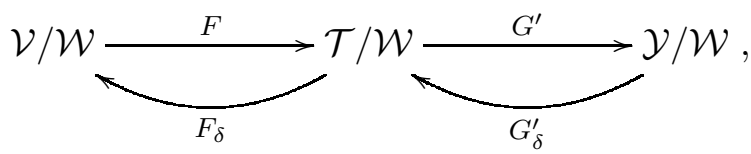

where $F$ and $G_{\delta}^{\prime}$ are the full embeddings.

Proof. Suppose that $F: \mathcal{V} / \mathcal{W} \rightarrow \mathcal{T} / \mathcal{W}$ and $G_{\delta}^{\prime}: \mathcal{Y} / \mathcal{W} \rightarrow \mathcal{T} / \mathcal{W}$ are the full embeddings. Let $F_{\delta}$ and $G^{\prime}$ be as in Lemma 3.5 and Lemma 3.6, respectively. Then $\left(F, F_{\delta}\right)$ and $\left(G^{\prime}, G_{\delta}^{\prime}\right)$ are adjoint pairs. Next we claim that $\operatorname{Im} F=\operatorname{Ker} G^{\prime}$. Obviously, $\operatorname{Im} F=\mathcal{V}$. For any $V \in \mathcal{V}$, there exists an $\mathbb{E}$-triangle

$$
V \longrightarrow Y_{V} \longrightarrow X_{V}-\rightarrow
$$

with $Y_{V} \in \mathcal{Y}$ and $X_{V} \in \mathcal{X}$. Since $\mathcal{T} \cap \mathcal{X}=\mathcal{V}$ and $\mathcal{X}$ is closed under extensions, $Y_{V} \in \mathcal{Y} \cap \mathcal{X}=\mathcal{W}$. Since $G^{\prime}(V)=Y_{V}, V \in \operatorname{Ker} G^{\prime}$ by Remark 2.6. Then $\operatorname{Im} F \subseteq \operatorname{Ker} G^{\prime}$. On the other hand, for any $T \in \operatorname{Ker} G^{\prime}, G^{\prime}(T)=Y_{T}$ appearing in an $\mathbb{E}$-triangle

$$
T \longrightarrow Y_{T} \longrightarrow X_{T}-\rightarrow
$$

$Y_{T} \in \mathcal{Y}$ and $X_{T} \in \mathcal{X}$. Then $Y_{T} \in \mathcal{W}$.

Applying the functor $\mathcal{C}(-, \mathcal{Y})$ to the $\mathbb{E}$-triangle $T \longrightarrow Y_{T} \longrightarrow X_{T} \rightarrow$, by Lemma 2.5 , we have the following exact sequence:

$$
\mathbb{E}\left(Y_{T}, \mathcal{Y}\right)=0 \rightarrow \mathbb{E}(T, \mathcal{Y}) \rightarrow \mathbb{E}\left(X_{T}, \Sigma \mathcal{Y}\right)=0
$$

It follows that $\mathbb{E}(T, \mathcal{Y})=0$ and then $T \in \mathcal{X}$. Thus $T \in \mathcal{T} \cap \mathcal{X}=\mathcal{V}$, namely, $T \in \operatorname{Im} F$. So $\operatorname{Im} F=\operatorname{Ker} G^{\prime}$. This completes the proof. 
Theorem 3.8. Let $(\mathcal{S}, \mathcal{T}),(\mathcal{U}, \mathcal{V})$ and $(\mathcal{X}, \mathcal{Y})$ be cotorsion pairs on $\mathcal{C}$ with $\mathcal{W}=\mathcal{S} \cap \mathcal{T}=$ $\mathcal{U} \cap \mathcal{V}=\mathcal{X} \cap \mathcal{Y}$. If $\mathcal{X} \cap \mathcal{T}=\mathcal{V}, \mathcal{Y} \subseteq \mathcal{T}, \Sigma \mathcal{V} \subseteq \mathcal{V}$ and $\Sigma \mathcal{Y} \subseteq \mathcal{Y}$, then there are two recollements of additive categories as follows:
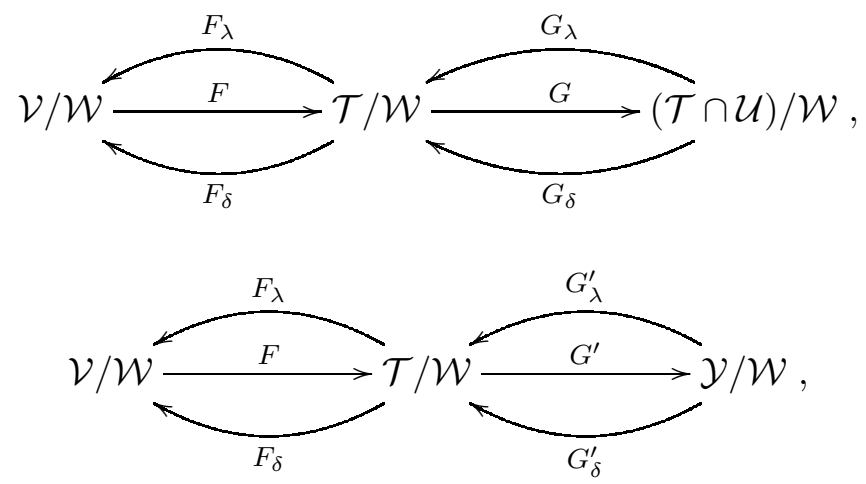

where $F, G_{\lambda}$ and $G_{\delta}$ are the full embeddings.

Proof. Define $J:(\mathcal{T} \cap \mathcal{U}) / \mathcal{W} \rightarrow \mathcal{Y} / \mathcal{W}$ in the following way. For any $M \in \mathcal{T} \cap \mathcal{U}, J(M)=Y_{M}$ appearing in an $\mathbb{E}$-triangle

$$
M \stackrel{y_{M}}{\longrightarrow} Y_{M} \longrightarrow X_{M} \rightarrow
$$

with $Y_{M} \in \mathcal{Y}$ and $X_{M} \in \mathcal{X}$. Applying the functor $\mathcal{C}\left(-, Y_{M^{\prime}}\right)$ to the above $\mathbb{E}$-triangle, we have the following exact sequence:

$$
\mathcal{C}\left(Y_{M}, Y_{M^{\prime}}\right) \stackrel{\mathcal{C}\left(y_{M}, W_{0}\right)}{\longrightarrow} \mathcal{C}\left(M, Y_{M^{\prime}}\right) \rightarrow \mathbb{E}\left(X_{M}, Y_{M^{\prime}}\right)=0
$$

Thus for any $f \in(\mathcal{T} \cap \mathcal{U}) / \mathcal{W})\left(M, M^{\prime}\right)$, there exists a morphism $f_{M} \in \mathcal{C}\left(Y_{M}, Y_{M^{\prime}}\right)$ such that $y_{M^{\prime}} \circ f=f_{M} \circ y_{M}$. Hence we have the following commutative diagram

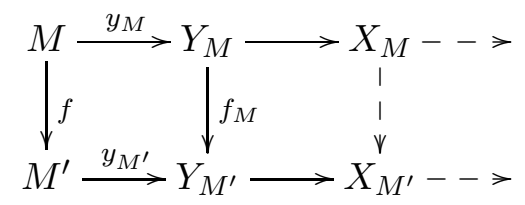

of $\mathbb{E}$-triangles. Define $J(f)=f_{M}$. By Lemma 2.11 and Lemma 2.12, $J$ is well defined and is an additive functor.

Define $K: \mathcal{Y} / \mathcal{W} \rightarrow(\mathcal{T} \cap \mathcal{U}) / \mathcal{W}$ in the following way. For any $Y \in \mathcal{Y}, K(Y)=U_{Y}$ appearing in an $\mathbb{E}$-triangle

$$
V_{Y} \longrightarrow U_{Y} \stackrel{u_{Y}}{\longrightarrow} Y \rightarrow
$$

with $V_{Y} \in \mathcal{V}$ and $U_{Y} \in \mathcal{U}$. Since $\mathcal{Y} \subseteq \mathcal{T}, \mathcal{V} \subseteq \mathcal{T}$ and $\mathcal{T}$ is closed under extensions, $U_{Y} \in \mathcal{T} \cap \mathcal{U}$. For any $g \in(\mathcal{Y} / \mathcal{W})\left(Y, Y^{\prime}\right)$, since $\mathbb{E}\left(U_{Y}, V_{Y^{\prime}}\right)=0$, there exists a morphism $g_{Y} \in \mathcal{C}\left(U_{Y}, U_{Y^{\prime}}\right)$ such that $u_{Y^{\prime}} \circ g_{Y}=g \circ u_{Y}$. Hence we have the following commutative diagram

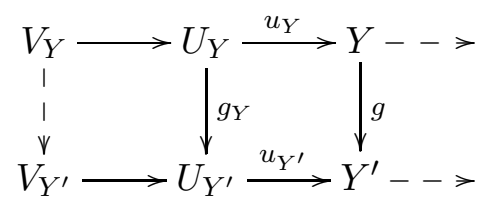


of $\mathbb{E}$-triangles. Define $K(g)=g_{Y}$. By Lemma 2.11 and Lemma 2.12, $K$ is well defined and is an additive functor. Now, we have the following diagram:

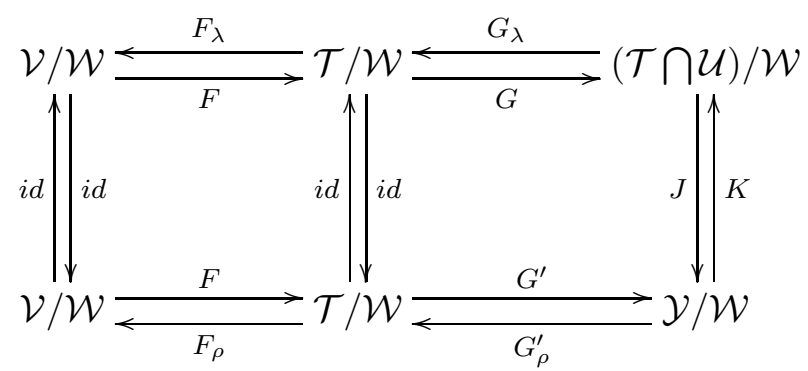

For any $Y \in \mathcal{Y}$, since $0 \rightarrow Y \rightarrow Y \rightarrow$ is an $\mathbb{E}$-triangle, we have $G^{\prime}(Y)=Y$. So $G^{\prime}$ is dense. Then there exists a natural isomorphism from the composite functor $\mathcal{T} / \mathcal{W} \stackrel{G^{\prime}}{\rightarrow} \mathcal{Y} / \mathcal{W} \stackrel{K}{\rightarrow}(\mathcal{T} \cap$ $\mathcal{U}) / \mathcal{W}$ to the functor $\mathcal{Y} / \mathcal{W} \stackrel{K}{\rightarrow}(\mathcal{T} \cap \mathcal{U}) / \mathcal{W}$. Consequently, we know that the composite functor $\mathcal{Y} / \mathcal{W} \stackrel{K}{\rightarrow}(\mathcal{T} \cap \mathcal{U}) / \mathcal{W} \stackrel{G_{\lambda}}{\rightarrow} \mathcal{T} / \mathcal{W}$ is indeed left adjoint to $\mathcal{T} / \mathcal{W} \stackrel{G^{\prime}}{\rightarrow} \mathcal{Y} / \mathcal{W}$. Put $G_{\lambda}^{\prime}=G_{\lambda} K$. Then we have a recollement of additive categories

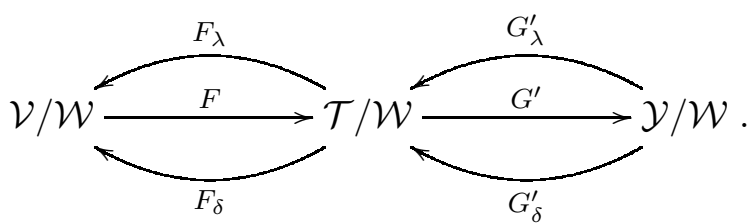

Similarly, for any $M \in \mathcal{T} \cap \mathcal{U}, G(M)=M$ since $0 \rightarrow W \rightarrow W \rightarrow$ is an $\mathbb{E}$-triangle. So $G$ is dense. Then there exists a natural isomorphism from the composite functor $\mathcal{T} / \mathcal{W} \stackrel{G}{\rightarrow}$ $(\mathcal{T} \cap \mathcal{U}) / \mathcal{W} \stackrel{J}{\rightarrow} \mathcal{Y} / \mathcal{W}$ to the functor $(\mathcal{T} \cap \mathcal{U}) / \mathcal{W} \stackrel{J}{\rightarrow} \mathcal{Y} / \mathcal{W}$. Thus $(\mathcal{T} \cap \mathcal{U}) / \mathcal{W} \stackrel{J}{\rightarrow} \mathcal{Y} / \mathcal{W} \stackrel{G_{\delta}^{\prime}}{\rightarrow} \mathcal{T} / \mathcal{W}$ is indeed right adjoint to $\mathcal{T} / \mathcal{W} \stackrel{G}{\rightarrow}(\mathcal{T} \cap \mathcal{U}) / \mathcal{W}$. Put $G_{\delta}=G_{\delta}^{\prime} J$. Then we obtain the desired recollement of additive categories

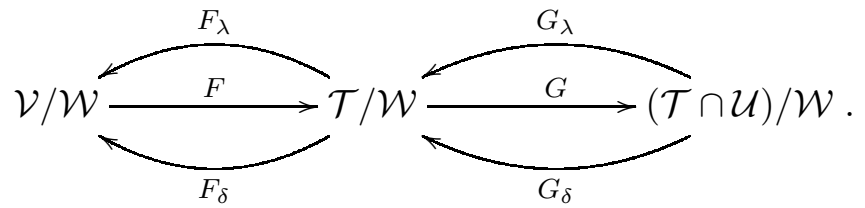

We give the dual result of Theorem [3.8, but omit its proof.

Theorem 3.9. Let $(\mathcal{S}, \mathcal{T}),(\mathcal{U}, \mathcal{V})$ and $(\mathcal{X}, \mathcal{Y})$ be cotorsion pairs on $\mathcal{C}$ with $\mathcal{W}=\mathcal{S} \cap \mathcal{T}=$ $\mathcal{U} \cap \mathcal{V}=\mathcal{X} \cap \mathcal{Y}$. If $\mathcal{Y} \cap \mathcal{S}=\mathcal{U}, \mathcal{X} \subseteq \mathcal{S}, \Sigma \mathcal{V} \subseteq \mathcal{V}$ and $\Sigma \mathcal{Y} \subseteq \mathcal{Y}$, then there are two recollements of additive categories as follows:

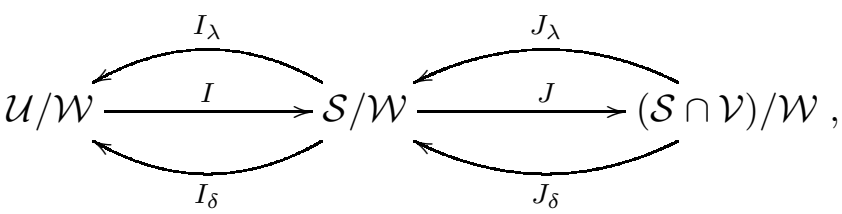




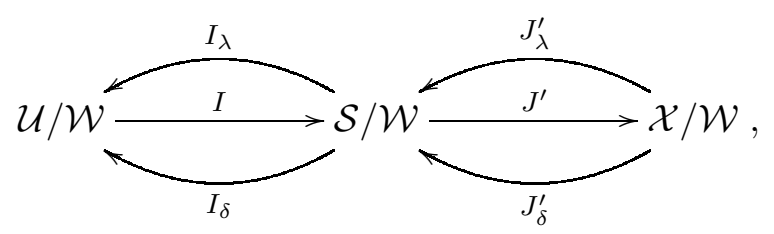

where $I, J_{\delta}$ and $J_{\lambda}^{\prime}$ are the full embeddings.

Remark 3.10. When we apply Theorem 3.8 to a triangulated category, it is just the Theorem 3.2 in [CLY]. When we apply Theorem 3.9 to a triangulated category, it is just the Theorem 3.9 in [CLY].

\section{Applications}

We assume that $R$ is a ring with unit. We denote by $\operatorname{Mod} R$ the category of left $R$-modules. Unless otherwise stated, all modules are left modules. In this section, we will give some applications. Meanwhile, we will see that Proposition 4.3 and Proposition 4.6 in [CLY] can be obtained directly from the cotorsion pairs on the categories of the complexes by our main results.

Let $\mathcal{A}$ be an abelian category with enough projective and injective objects. For a subclass $\mathcal{C}$ of $\mathcal{A}$, we set

$$
\begin{aligned}
{ }^{\perp} \mathcal{C} & =\left\{M \in \mathcal{A} \mid \operatorname{Ext}_{R}^{1}(M, N)=0 \text { for any } N \in \mathcal{C}\right\} \\
\mathcal{C}^{\perp} & =\left\{N \in \mathcal{A} \mid \operatorname{Ext}_{R}^{1}(M, N)=0 \text { for any } M \in \mathcal{C}\right\} .
\end{aligned}
$$

A pair $(\mathcal{X}, \mathcal{Y})$ of $\mathcal{A}$ is said to be a cotorsion pair if $\mathcal{X}={ }^{\perp} \mathcal{Y}$ and $\mathcal{Y}=\mathcal{X}^{\perp}$. The cotorsion pair is called complete if for any $M \in \mathcal{A}$, there exist two short exact sequences

$$
\begin{aligned}
& 0 \rightarrow Y \rightarrow X \rightarrow M \rightarrow 0 \\
& 0 \rightarrow M \rightarrow Y^{\prime} \rightarrow X^{\prime} \rightarrow 0
\end{aligned}
$$

with $X, X^{\prime} \in \mathcal{X}$ and $Y, Y^{\prime} \in \mathcal{Y}$. A pair of cotorsion pairs $((\mathcal{S}, \mathcal{T}),(\mathcal{U}, \mathcal{V})$ is said to be a twin cotorsion pair if $\mathcal{S} \subseteq \mathcal{U}$, or equivalently, $\mathcal{V} \subseteq \mathcal{T}$. A twin cotorsion pair is called concentric if $\mathcal{S} \cap \mathcal{T}=\mathcal{U} \cap \mathcal{V}$.

It is well-known that the canonical cotorsion pair $(\mathcal{P}, \operatorname{Mod} R)$ is completed by $G \mathrm{GT}$, Theorem 3.2 .1 since it is generated by $R$.

Recall that $R$ is said to be a Gorenstein ring if it is a left and right noetherian ring with finite injective dimension on either side. An $R$-module $N$ is called Gorenstein projective if there is a exact sequence of projective $R$-modules

$$
\mathbf{P}: \quad \cdots \rightarrow P_{1} \rightarrow P_{0} \rightarrow P^{0} \rightarrow P^{1} \rightarrow \cdots
$$

with $N=\operatorname{Ker}\left(P_{0} \rightarrow P^{0}\right)$ such that $\operatorname{Hom}(\mathbf{P}, Q)$ is exact for any projective module $Q$.

Let $\mathcal{G P}(R)$ be the full subcategory of $\operatorname{Mod} R$ consisting of all the Gorenstein projective module. We denote by $\mathcal{P}^{<\infty}$ the class of modules admitting finite projective dimension. If $R$ is 
a Gorenstein ring, then $\left(\mathcal{G P}(R), \mathcal{P}^{<\infty}\right)$ is a complete cotorsion pair, see [GT, Example 4.1.14].

Proposition 4.1. Let $R$ be a Gorenstein ring. Then the stable category $\mathcal{G P}(R)$ is a coreflection subcategory of $\underline{\operatorname{Mod} R}$.

Proof. It is easy to see that $\left((\mathcal{P}, \operatorname{Mod} R),\left(\mathcal{G P}(R), \mathcal{P}^{<\infty}\right)\right)$ is a concentric twin cotorsion pair and $\Sigma \mathcal{P}^{<\infty} \subseteq \mathcal{P}^{<\infty}$ since $R$ is a Gorenstein ring. By Lemma 3.4, we obtain a colocalization sequence of additive categories

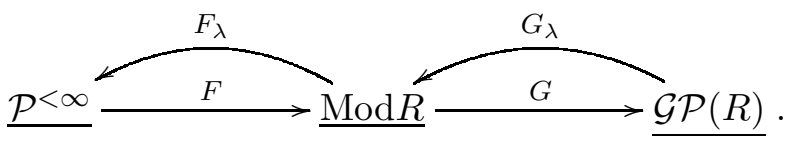

Thus, the inclusion functor $G_{\lambda}$ admits a right adjoint functor. That is, $\mathcal{G P}(R)$ is a coreflection subcategory of $\underline{\operatorname{Mod} R}$.

We denote the class of projective modules by $\mathcal{P}$, the class of injective modules by $\mathcal{I}$, the the category of a complex of left $R$-modules by $C(\operatorname{Mod} R)$, the class of exact complexes by $\mathcal{E}$.

We write a complex $X^{\bullet} \in C(\operatorname{Mod} R)$ as

$$
\cdots \longrightarrow X_{n+1} \stackrel{d_{n+1}}{\longrightarrow} X_{n} \stackrel{d_{n}}{\longrightarrow} X_{n-1} \longrightarrow \cdots .
$$

Given $X^{\bullet} \in C(\operatorname{Mod} R)$, the suspension of $X^{\bullet}$, denoted by $S\left(X^{\bullet}\right)$, is the complex given by $S\left(X^{\bullet}\right)_{n}=X_{n-1}$ and $d_{S\left(X^{\bullet}\right)}=-d_{n}$. Inductively, one can define $S^{n+1}\left(X^{\bullet}\right)=S\left(S^{n}\left(X^{\bullet}\right)\right)$ for all $n \in \mathbb{Z}$. Given an $R$-module $X$, we denote by $\bar{X}$ the complex

$$
\cdots \longrightarrow 0 \longrightarrow X \stackrel{1}{\longrightarrow} X \longrightarrow 0 \longrightarrow \cdots
$$

where the two $X$ 's are in the 0th and -1st place.

Recall that a complex $P^{\bullet} \in C(\operatorname{Mod} R)$ is said to be projective if for any morphism $P^{\bullet} \rightarrow D^{\bullet}$ and any epimorphism $C^{\bullet} \rightarrow D^{\bullet}$, the diagram

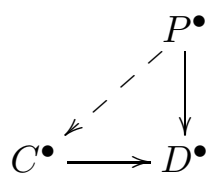

can be completed to a commutative diagram by a morphism $P^{\bullet} \rightarrow C^{\bullet}$. Dually, one can define the injective complex $I^{\bullet}$. It is well-known that each projective complex $P^{\bullet}$ and injective complex $I^{\bullet}$ are exact complexes. Moreover, the components $P_{n}, I_{n}$ of projective complex $P^{\bullet}$ and injective complex $I^{\bullet}$ are projective and injective modules in $\operatorname{Mod} R$, respectively. However, conversely, it may not true, see [EJ, Example 1.4.5].

From [EJ, Section 1.4], we know that $C(\operatorname{Mod} R)$ admits enough projective and injective complexes. Then, for any complex $C^{\bullet} \in C(\operatorname{Mod} R)$, there exist a projective resolution and an injective resolution of $C^{\bullet}$. It means that there exist two exact sequences of complexes

$$
\cdots \longrightarrow P^{\bullet}{ }_{n} \longrightarrow \cdots \longrightarrow P_{1}^{\bullet} \longrightarrow P_{0}^{\bullet} \longrightarrow C^{\bullet} \longrightarrow 0
$$




$$
0 \longrightarrow C^{\bullet} \longrightarrow I_{0}^{\bullet} \longrightarrow I_{-1}^{\bullet} \longrightarrow \cdots \longrightarrow I_{-n}^{\bullet} \longrightarrow \cdots
$$

where each $P_{n}^{\bullet}, I_{-n}^{\bullet}$ is projective and injective, respectively.

Now, we can define the groups $\operatorname{Ext}_{C(\operatorname{Mod} R)}^{n}\left(C^{\bullet}, D^{\bullet}\right)$ (or simply, $\left.\operatorname{Ext}^{n}\left(C^{\bullet}, D^{\bullet}\right)\right)$ for any complexes $C^{\bullet}$ and $D^{\bullet}$. If exact sequences of complexes

$$
\cdots \longrightarrow P_{n}^{\bullet} \longrightarrow \cdots \longrightarrow P_{1}^{\bullet} \longrightarrow P_{0}^{\bullet} \longrightarrow C^{\bullet} \longrightarrow 0
$$

is the projective resolution of $C^{\bullet}$, then $\operatorname{Ext}^{n}\left(C^{\bullet}, D^{\bullet}\right)$ is defined to be the $n$-cohomology group of the complex

$$
0 \rightarrow \operatorname{Hom}\left(P_{0}^{\bullet}, D^{\bullet}\right) \rightarrow \operatorname{Hom}\left(P_{1}^{\bullet}, D^{\bullet}\right) \rightarrow \cdots .
$$

It also can be computed by the injective resolution of $D^{\bullet}$. Especially, the $\xi \in \operatorname{Ext}^{1}\left(C^{\bullet}, D^{\bullet}\right)$ can be put in bijective correspondence with the equivalence classes of short exact sequences

$$
0 \longrightarrow D^{\bullet} \longrightarrow E^{\bullet} \longrightarrow C^{\bullet} \longrightarrow 0
$$

in $C(\operatorname{Mod} R)$.

We denote by $\mathbf{K}(\operatorname{Mod} R)$ and $\mathbf{D}(R)$ the corresponding homotopy category and derived category of $\operatorname{Mod} R$.

Definition 4.2. G01, G02 Given a class of $R$-modules $\mathcal{C}$, we define the following classes of chain complexes in $C(\operatorname{Mod} R)$

(1) $d w \mathcal{C}$ is the class of all chain complexes $C^{\bullet}$ with $C_{n} \in \mathcal{C}$ for any $n \in \mathbb{Z}$.

(2) exC is the class of all exact chain complexes $C^{\bullet}$ with $C_{n} \in \mathcal{C}$ for any $n \in \mathbb{Z}$.

(3) $\widetilde{\mathcal{C}}$ is the class of all exact chain complexes $C^{\bullet}$ with cycles $Z_{n}\left(C^{\bullet}\right) \in \mathcal{C}$.

(4) Given any cotorsion pair $(\mathcal{X}, \mathcal{Y})$ in $\operatorname{Mod} R . d g \mathcal{X}$ is the class of all complexes of $R$-modules satisfying that each $X_{n} \in \mathcal{X}$ and $\operatorname{Hom}_{\mathbf{K}(\operatorname{Mod} R)}\left(X^{\bullet}, Y^{\bullet}\right)=0$ for any $X^{\bullet} \in d g \mathcal{X}$ and any $Y^{\bullet} \in \tilde{\mathcal{Y}}$. Similarly, one can define $d g \mathcal{Y}$.

We denote by $\mathbf{K}_{e x}(\mathcal{C})$ and $\mathbf{K}(\mathcal{C})$ the corresponding homotopy categories consisting of complexes in $e x \mathcal{C}$, and $d w \mathcal{C}$, respectively.

Lemma 4.3. [CLY, G03] For the canonical cotorsion pair $(\mathcal{P}, \operatorname{Mod} R),\left(d w \mathcal{P}, d w \mathcal{P}^{\perp}\right),(e x \mathcal{P}$, ex $\left.\mathcal{P}^{\perp}\right)$ and $(d g \mathcal{P}, \mathcal{E})$ are the complete cotorsion pairs in $C(\operatorname{Mod} R)$. Moreover, they satisfy the following conditions:

(1) $\widetilde{\mathcal{P}}=d w \mathcal{P} \bigcap d w \mathcal{P}^{\perp}=e x \mathcal{P} \bigcap e x \mathcal{P}^{\perp}=d g \mathcal{P} \cap \mathcal{E}$

(2) $\mathcal{E} \bigcap d w \mathcal{P}=e x \mathcal{P}, d g \mathcal{P} \subseteq d w \mathcal{P}$.

Lemma 4.4. The classes ex $\mathcal{P}^{\perp}$ and $\mathcal{E}$ are closed under cosyzygy $\Sigma$. 
Proof. Let $M^{\bullet} \in \mathcal{E}$ and $N^{\bullet} \in e x \mathcal{P}^{\perp}$. Since $C(\operatorname{Mod} R)$ admits enough injective objects. Then, there exist two short exact sequence

$$
\begin{gathered}
0 \longrightarrow M^{\bullet} \longrightarrow E^{\bullet}\left(M^{\bullet}\right) \rightarrow \Sigma M^{\bullet} \longrightarrow 0 \\
0 \longrightarrow N^{\bullet} \longrightarrow E^{\bullet}\left(N^{\bullet}\right) \rightarrow \Sigma N^{\bullet} \longrightarrow 0
\end{gathered}
$$

with $E^{\bullet}\left(M^{\bullet}\right)$ and $E^{\bullet}\left(N^{\bullet}\right)$ are injective complexes. It is easy to check that $\Sigma M^{\bullet}$ by the long exact sequence theorem and injective complexes are exact. Hence, $\mathcal{E}$ is closed under cosyzygy.

Now, it remains to show that $\Sigma N^{\bullet} \in e x \mathcal{P}^{\perp}$. Let $Q^{\bullet}$ be a complex of exP. Applying the functor $\operatorname{Hom}\left(Q^{\bullet},-\right)$ to the exact sequence (4.1), we obtain a long exact sequence

$$
\operatorname{Ext}^{1}\left(Q^{\bullet}, N^{\bullet}\right) \longrightarrow \operatorname{Ext}^{1}\left(Q^{\bullet}, E^{\bullet}\left(N^{\bullet}\right)\right) \longrightarrow \operatorname{Ext}^{1}\left(Q^{\bullet}, \Sigma N^{\bullet}\right) \longrightarrow \operatorname{Ext}^{2}\left(Q^{\bullet}, N^{\bullet}\right)
$$

Note that there is a short exact sequence

$$
0 \longrightarrow \Omega Q^{\bullet} \longrightarrow P^{\bullet} \longrightarrow N^{\bullet} \longrightarrow 0
$$

Thus, we know that $\operatorname{Ext}^{2}\left(Q^{\bullet}, N^{\bullet}\right) \cong \operatorname{Ext}^{1}\left(\Omega Q^{\bullet}, N^{\bullet}\right)$. We claim that $\Omega Q^{\bullet} \in \operatorname{exP}$. Indeed, it is easy to see that $\Omega Q^{\bullet}$ is exact. Moreover, the sequence (4.2) is degree-wise split. Hence, each $Q_{n}$ is a projective module and so, the desired result comes. In this case, $\operatorname{Ext}^{2}\left(Q^{\bullet}, N^{\bullet}\right)=$ $\operatorname{Ext}^{1}\left(Q^{\bullet}, E^{\bullet}\left(N^{\bullet}\right)\right)=0$. Therefore, $\operatorname{Ext}^{1}\left(Q^{\bullet}, \Sigma N^{\bullet}\right)=0$. This completes the proof.

Now, we can apply one of our main results to reprove the existence of the following recollement.

Proposition 4.5. CLY] There exists the following recollement of triangulated category

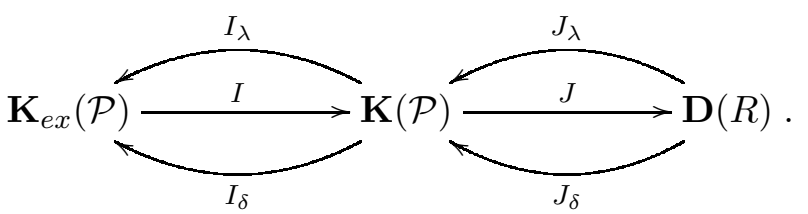

Proof. By Theorem 3.9, Lemma 4.3 and Lemma 4.4, we have the following recollement

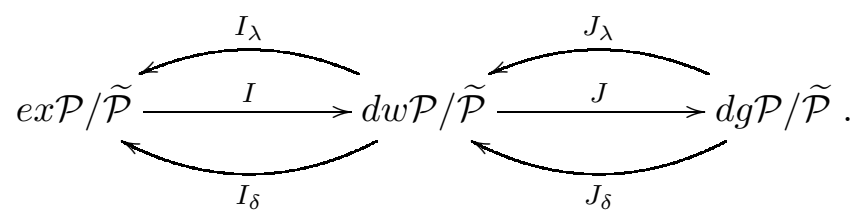

It is well-known that there exist triangulated equivalences $\operatorname{ex} \mathcal{P} / \widetilde{\mathcal{P}} \cong \mathbf{K}_{e x}(\mathcal{P}), d w \mathcal{P} / \widetilde{\mathcal{P}} \cong \mathbf{K}(\mathcal{P})$ and $d g \mathcal{P} / \widetilde{\mathcal{P}} \cong \mathbf{D}(R)$. Moreover, it is easy to see that the functor $I$ and $J_{\lambda}$ are triangulated functors. By adjointness, we know that the remained four functors are triangulated functors.

Next, we hope to get the Krause's recollement from the cotorsion pair on the category of complexes. The following observation is very important. Let $X^{\bullet}$ be a complex. Now, we 
construct a short exact of complexes as follows.

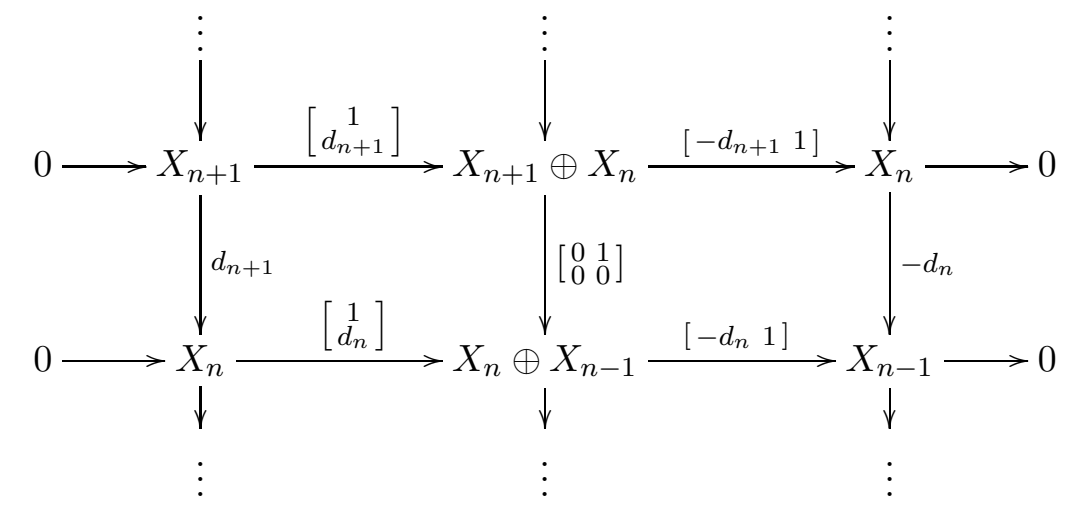

That is, there exists an exact sequence

$$
0 \longrightarrow X \bullet \stackrel{\left[\begin{array}{l}
1 \\
d
\end{array}\right]}{\longrightarrow} \prod_{n \in \mathbb{Z}} \overline{X_{n}} \stackrel{\left[\begin{array}{ll}
-d & 1
\end{array}\right]}{\longrightarrow} S(X) \longrightarrow 0 .
$$

From this observation, we have the following result.

Lemma 4.6. If $X^{\bullet} \in d g \mathcal{I}$, then there exists an isomorphism of complexes $\Sigma X^{\bullet} \cong S(X)$. In particularly, $d g \mathcal{I}$ is closed under the cosyzygy $\Sigma$.

Proof. Since $X^{\bullet} \in d g \mathcal{I}, X_{n}$ are injective modules for all $n \in \mathbb{Z}$. Note that $\prod_{n \in \mathbb{Z}} \overline{X_{n}}$ is an injective complex since $\overline{X_{n}}$ are injective complexes for all $n \in \mathbb{Z}$. Then we obtain the desired result.

Finally, we are in proposition to reobtain the Krause's recollement, which also reprove the Proposition 4.3 in CLY].

Proposition 4.7. For the canonical cotorsion pair $(\operatorname{Mod} R, \mathcal{I}),\left({ }^{\perp} d w \mathcal{I}, d w \mathcal{I}\right),\left({ }^{\perp}\right.$ exI , exI $)$ and $(\mathcal{E}, d g \mathcal{I})$ are the completed cotorsion pairs in $C(\operatorname{Mod} R)$. Moreover, they induces Krause's recollement

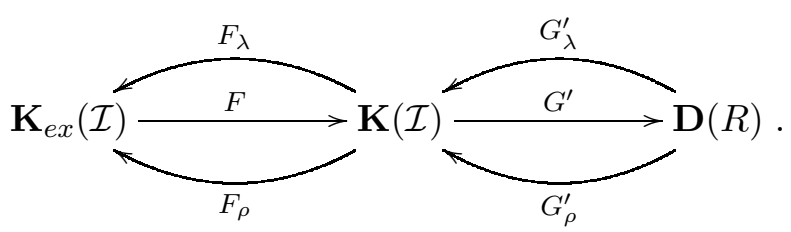

Proof. Following the references [CLY, G03], we know that these three cotorsion pairs are complete and they also satisfy the following conditions:

(1) $\widetilde{\mathcal{I}}={ }^{\perp} d w \mathcal{I} \cap d w \mathcal{I}={ }^{\perp} e x \mathcal{I} \cap e x \mathcal{I}=\mathcal{E} \cap d g \mathcal{I}$

(2) $\mathcal{E} \cap d w \mathcal{I}=e x \mathcal{I}, d g \mathcal{I} \subseteq d w \mathcal{I}$.

Now, we show that $e x \mathcal{I}$ is closed under cosyzygy. Indeed, there exists a short exact sequence $0 \longrightarrow X^{\bullet} \longrightarrow E^{\bullet}\left(X^{\bullet}\right) \longrightarrow \Sigma X^{\bullet} \longrightarrow 0$. Clearly, $\Sigma X^{\bullet}$ is an exact complex. It is easy to see 
that this sequence is degree-wise split. Thus, each component $\left(\Sigma X^{\bullet}\right)_{n}$ of $\Sigma X^{\bullet}$ is injective and so, $\Sigma X^{\bullet} \in e x \mathcal{I}$.

By Lemma 4.6, $d g \mathcal{I}$ is also closed under the cosyzygy $\Sigma$. Therefore, by Theorem 3.8, we know that there exists a recollement of additive categories

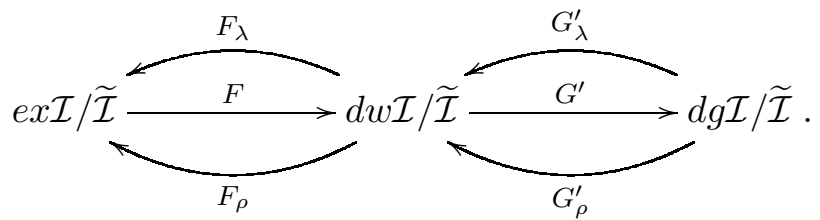

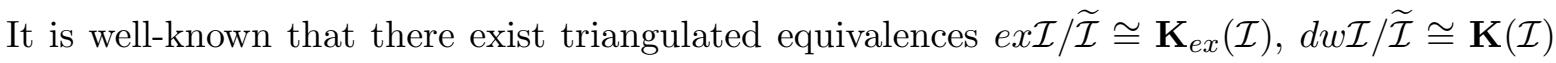
and $d g \mathcal{I} / \widetilde{\mathcal{I}} \cong \mathbf{D}(R)$. The remaining arguments are similar to Proposition 4.5,

\section{References}

[B] F. Borceux. Handbook of categorical algebra, Volume 1. Basic category theory. Encyclopedia of Mathematics and its Applications, 50. Cambridge University Press, Cambridge, 1994.

[BBD] A. Beilinson, J. Bernstein, P. Deligne. Faisceaux pervers. (French) [Perverse sheaves] Analysis and topology on singular spaces, I (Luminy, 1981), 5-171, Astérisque, 100, Soc. Math. France, Paris, 1982.

[CLY] W. Chen, Z. Liu, X. Yang. Recollements associated to cotorsion pairs. J. Algebra Appl. 17(5): 1-15, 2018.

[EJ] E. E. Enochs, and O. M. G. Jenda, Relative Homological Algebra. Volume 2. Berlin, Boston: De Gruyter, 2011.

[GT] R. Göbel and J. Trlifaj, Approximations and endomorphism algebras of modules, Walter de Gruyter, Berlin, 2006.

[G01] J. Gillespie, The flat model structure on Ch(R), Trans. Amer. Math. Soc. 356(8): 33693390, 2004.

[G02] J. Gillespie, Cotorsion pairs and degreewise homological model structures, Homology, Homotopy Appl. 10(1): 283-304, 2008.

[G03] J. Gillespie, Gorenstein complexes and recollements from cotorsion pairs, Adv. Math. 291: 859-911, 2016.

[LN] Y. Liu, H. Nakaoka. Hearts of twin cotorsion pairs on extriangulated categories. J. Algebra 528: 96-149, 2019.

[MV] R. MacPherson, K. Vilonen. Elementary construction of perverse sheaves. Invent. Math. 84(2): 403-435, 1986. 
[NP] H. Nakaoka, Y. Palu. Extriangulated categories, Hovey twin cotorsion pairs and model structures. Cah. Topol. Gom. Diffr. Catg. 60(2): 117-193, 2019.

[SS] J. Šaroch, J. Šťovíček, Singular compactness and definability for $\Sigma$-cotorsion and Gorenstein modules, arXiv :1804.09080.

[WL] M. Wang, Z. Lin. Recollement of additive quotient categories. arXiv:1502.00479, 2015.

[ZW] Q. Zheng, J. Wei. One-sided triangulated categories induced by concentric twin cotorsion pairs. To appear in J. Algebra Appl. DOI: 10.1142/S021949882050142X.

[ZZ] P. Zhou, B. Zhu, Triangulated quotient categories revisited. J. Algebra. 502: 196-232, 2018.

[ZhZ] B. Zhu B, X. Zhuang. Tilting subcategories in extriangulated categories. arXiv:1907.00747, 2019.

Yonggang $\mathrm{Hu}^{a, c}$ and Panyue $\mathrm{Zhou}^{b, c}$

${ }^{a}$ College of Applied Sciences, Beijing University of Technology, 100124 Beijing, P. R. China.

${ }^{b}$ College of Mathematics, Hunan Institute of Science and Technology, 414006, Yueyang, Hunan, P. R. China.

${ }^{c}$ Département de Mathématiques, Université de Sherbrooke, Sherbrooke, Québec J1K 2R1, Canada.

Yonggang $\mathrm{Hu}$ E-mail: huyonggang@emails.bjut.edu.cn

Panyue Zhou E-mail: panyuezhou@163.com 